\title{
Estudio Comparativo de la Efectividad Antiinflamatoria Postoperatoria al usar Dexametasona Endovenoso Versus Ketoprofeno Endovenoso previo a la exodoncia de terceros Molares Mandibulares Incluidos
}

\author{
Comparative Study of Postoperative Anti-inflammatory Effectiveness when Using \\ intravenous Dexamethasone Versus Intravenous Ketoprofen Prior to Extraction \\ of Mandibular third Molars Included
}

Cristhian Collazos Peña'; Diego Fonseca Escobar'; Fernando Parada Fernández ${ }^{1}$ \& Sandra Montero Riffo²

COLLAZOS, P. C.; FONSECA. E. D.; PARADA, F. F. \& MONTERO, R. S. Estudio comparativo de la efectividad antiinflamatoria postoperatoria al usar Dexametasona endovenoso versus Ketoprofeno endovenoso previo a la exodoncia de terceros molares mandibulares incluidos. Int. J. Odontostomat., 15(4):1060-1070, 2021.

RESUMEN: La exodoncia de terceros molares incluidos conlleva la mayoría de las veces un cuadro inflamatorio agudo, dolor postoperatorio y trismus. En la actualidad, se han propuesto diversos protocolos farmacológicos con el fin de prevenir estas complicaciones, donde los más utilizados son los analgésicos y corticoides. Comparar Dexametasona y Ketoprofeno endovenoso previo a la cirugía de terceros molares mandibulares incluidos, en el control del edema, dolor y trismus. Se realizó un estudio experimental, analítico de corte transversal, autorizado por el comité de ética de la Universidad Andrés Bello. Se escogieron 30 sujetos que requerían exodoncia de terceros molares mandibulares incluidos, de forma aleatoria 15 sujetos recibieron Dexametasona $4 \mathrm{mg}$ endovenoso y 15 Ketoprofeno $200 \mathrm{mg}$ endovenoso 30 minutos antes de la intervención. El procedimiento quirúrgico fue estandarizado, se evaluó el edema facial, trismus y dolor postquirúrgico a los 2 y 7 días terminada la cirugía. Se presentó una diferencia estadísticamente significativa solo en 2 mediadas faciales, de las 5 tomadas, al comparar el porcentaje de edema a los 7 días postoperatorias entre ambos grupos experimentales. La mayoría del grupo Dexametasona presentó un edema de mayor volumen en comparación al grupo Ketoprofeno. En relación al dolor y trismus, sólo se observan diferencias significativas en la intensidad del dolor evaluada a los 7 días, siendo mayor en el grupo Ketoprofeno. En las otras variables medidas, la presencia de odontosección en la cirugía generó una diferencia estadísticamente significativa, siendo mayor el edema en los que se les realizó odontosección. Esta diferencia se vio principalmente en el grupo Ketoprofeno evaluado a los 2 días postoperatorios. El uso de cualquiera de los 2 fármacos está bien indicado para aliviar el dolor e inflamación en este tipo de cirugía. Pese a que el grupo tratado con Dexametasona presentó menor dolor, no fue una diferencia significativa en comparación al grupo con Ketoprofeno.

PALABRAS CLAVE: dexametasona, ketoprofeno, edema, tercer molar, trismus.

\section{INTRODUCCIÓN}

Son múltiples las razones por las que los terceros molares se encuentran incluidos, una de estas puede deberse a la interacción anormal de tejidos dentarios durante su desarrollo, lo que provocaría la erupción de un diente ectópico (Büyükkurt et al., 2005) o simplemente una falta de espacio en la arcada dentaria al ser los últimos en erupcionar. Los terceros molares incluidos corresponden a una de las patologías más frecuentes encontradas en el territorio maxilofacial (Salmerón et al., 2008). Éstos se categorizan de acuerdo a su posición y profundidad en relación al segundo molar, su proximidad con la rama ascendente de la mandíbula y su angulación hacia el segundo molar adyacente, siendo los terce-

\footnotetext{
${ }^{1}$ Cirujano Dentista - Universidad Andrés Bello, Facultad de Odontología, Santiago de Chile.

${ }^{2}$ Cirujano Bucomaxilofacial - Universidad Andrés Bello, Facultad de Odontología, Santiago de Chile.
}

Received: 2021-05-22 Accepted: 2021-06-23 
COLLAZOS, P. C.; FONSECA. E. D.; PARADA, F. F. \& MONTERO, R. S. Estudio comparativo de la efectividad antiinflamatoria postoperatoria al usar Dexametasona endovenoso versus Ketoprofeno endovenoso previo a la exodoncia de terceros molares mandibulares incluidos. Int. J. Odontostomat., 15(4):1060-1070, 2021.

ros molares impactados mesioangularmente los más prevalentes en el arco mandibular (García et al., 2007).

El acto quirúrgico al extraer los terceros molares incluidos es una práctica no exenta de complicaciones, ya sean, neuropáticas, inflamatorias e infecciosas. La exodoncia produce casi siempre un cuadro inflamatorio agudo, dolor postoperatorio y trismus, que generalmente afecta en la calidad de vida del paciente (Gay-Escoda \& Berini, 2004). La agresión a los tejidos blandos orales y periorales, incisiones propias de la cirugía, el decolado del colgajo, el grado de traumatismo óseo y dental, son los responsables de la intensidad de los signos y/o síntomas inflamatorios que se generan al paciente después de la cirugía (Grossi et al., 2007). En condiciones normales la inflamación, no se observan inmediatamente después de la cirugía, sino que comienza gradualmente alcanzando su máxima expresión entre el segundo día (48 horas) y tercer día (72 horas) postoperatorios (Grossi et al.). Diversos estudios actuales se han orientado en poder combatir las complicaciones de este tipo de cirugías y conseguir un mejor control postoperatorio que surge en los pacientes. Es por esto que se han propuesto varias combinaciones farmacológicas y algunos procedimientos técnicos que intentan disminuir las secuelas indeseadas. Es sabido que el mejor tratamiento preventivo de la inflamación consiste en la realización de una cirugía técnicamente correcta, rápida, limpia y lo menos traumática posible, aplicando los productos más inocuos y efectivos que nos brinda la farmacología (Prieto et al., 2005).

De las numerosas opciones farmacológicas disponibles encontramos los antiinflamatorios esteroidales, también llamados corticoides. Estos son un potente modulador de la inflamación, actuando mediante la inhibición de la fosfolipasa A2, enzima responsable de la inducción de ácido araquidónico obtenido de la membrana celular. Con una disminución de esta enzima, se inhibe la vasodilatación vascular, reduciendo la transudación de líquidos, por ende, la formación de edema (Herrera-Briones et al., 2013), a la vez actúa inhibiendo mediadores químicos de la inflamación lo que reduce los signos y/o síntomas inflamatorios postoperatorios (Moraschini et al., 2016; Kumar et al., 2021). La farmacología actual dispone de corticoides con un potencial antiinflamatorio variable.

Estos últimos años ha adquirido mayor importancia la dexametasona, debido a su acción glucocorticoide y no mineralocorticoide, disminuyendo los efectos adversos del consumo crónico de corticoides.
Los glucocorticoides inhiben la liberación de hormona Adenocorticotrofina (ACTH), lo que tras su uso continuado genera una atrofia del tejido suprarrenal (Prieto et al.). El uso clínico de este tipo de medicamentos debe ser moderado y racional, por tiempo y dosis limitadas, ya que, de acuerdo con los análisis endocrinólogos, después del quinto día de uso, la terapia comienza a producir inmunosupresión, condición que en algunos pacientes puede tomar hasta 9 meses para volver a los niveles normales (Laureano Filho et al., 2008; de la Cruz Carranza et al., 2013) Sin embargo, la Dexametasona ha demostrado ser un medicamento de administración segura, en tiempo y dosis adecuadas (Laureano Filho et al.). Las vías de administración son diversas, y con resultados muy variables, en donde se encuentran descritas principalmente las vías parenterales, siendo una de ellas la vía endovenosa, la cual nos otorga rápidamente buenas concentraciones del fármaco y, por ende, genera una acción antiinflamatoria prolongada (Majid \& Mahmood, 2011). Dado las reacciones adversas que poseen los glucocorticoides y el miedo del uso por parte del odontólogo gracias a la falta de control terapéutico, cabe la necesidad de observar otra terapia farmacológica que nos ayude a controlar la inflamación, trismus y dolor postoperatorio en la exodoncia de terceros molares mandibulares, es aquí donde encontramos a los antiinflamatorios no esteroidales (AINES) los cuales en dosis adecuadas, corresponden a una buena opción para combatir estas complicaciones. La mayoría de los AINES actúan principalmente inhibiendo la enzima ciclooxigenasa (COX), previniendo la síntesis local de prostaglandinas. Actualmente los AINES empleados inhiben el efecto de las dos isoformas de la enzima, COX-1 y COX-2 (Esteller et al., 2004).

Uno de los antiinflamatorios no esteroidales que forma parte de este grupo es el Ketoprofeno, el cual se emplea para el tratamiento de enfermedades reumáticas, traumatologías y procesos inflamatorios en general. Puede administrarse vía oral (50 o $200 \mathrm{mg}$ ) o parenteral (100 mg intramuscular y endovenoso). El Ketoprofeno es derivado del ácido propiónico, el cual muestra actividad antiinflamatoria (a altas dosis), analgésica (a dosis bajas) y antipirética. Muchas de sus acciones parecen estar asociadas principalmente con la inhibición de las ciclooxigenasas con la consecuencia reducción de la producción de las prostaglandinas (Esteller et al.). También es un potente inhibidor de la bradicinina, uno de los mediadores químicos de la inflamación y del dolor, y previene la liberación de enzimas lisosomales que provocan la destrucción tisular en las reacciones inflamatorias 
COLLAZOS, P. C.; FONSECA. E. D.; PARADA, F. F. \& MONTERO, R. S. Estudio comparativo de la efectividad antiinflamatoria postoperatoria al usar Dexametasona endovenoso versus Ketoprofeno endovenoso previo a la exodoncia de terceros molares mandibulares incluidos. Int. J. Odontostomat., 15(4):1060-1070, 2021.

(Buesa Martínez, 1995). El Ketoprofeno como todos los AINES, puede provocar úlceras, hemorragias, o perforaciones gastrointestinales, por lo que se contraindica su uso en personas con estos antecedentes, además de pacientes con predisposición hemorrágica, insuficiencia hepática grave, insuficiencia renal grave, tercer trimestre de gestación, insuficiencia cardiaca grave, cualquier tipo de trastornos hemostáticos o en tratamiento con anticoagulantes (Weinberg \& Froum, 2014). Debido a la escasa evidencia científica disponible que compare cuál de estos dos fármacos, Dexametasona y Ketoprofeno, es más eficaz en el control de la inflamación y trismus postoperatorio, nace la necesidad de este estudio, el cual busca determinar si existe diferencia tanto en el edema, dolor y apertura bucal en el día dos y siete postoperatorios. En pacientes premedicados, un grupo con Dexametasona y otro con Ketoprofeno, vía endovenosa, tomando como grupo control los pacientes sanos antes de someterse a la cirugía, con el fin de establecer cuál de los dos fármacos presenta mejor acción en contra de estas complicaciones y determinar el uso/beneficio que sea más conveniente.

\section{MATERIAL Y MÉTODO}

Se realizó un estudio clínico experimental, analítico de corte transversal en las instalaciones de la clínica odontológica de la Universidad Andrés Bello, sede Santiago, con los pacientes atendidos en el postgrado de Cirugía Maxilofacial, en donde se evaluó a un universo de 30 pacientes sanos, sin patologías crónicas y agudas, de ambos sexos, mayores de 18 años, que fueron sometidos a exodoncia de terceros molares mandibulares incluidos que necesitaban osteotomía, en el periodo académico de Julio a Octubre y que voluntariamente aceptaron ser parte del presente estudio mediante la firma de un consentimiento informado. Se excluyeron los sujetos que no aceptaron participar o se perdieron en el transcurso de la investigación, pacientes que no se logró colocar la vía endovenosa, los alérgicos a los fármacos a usar, gestantes o mujeres en período de lactancia y sujetos que hubiesen tomado algún fármaco antiinflamatorio en las últimas 24 horas previo a la cirugía.

Los grupos experimentales fueron obtenidos de forma probabilístico y aleatorio simple, en la cual la muestra de 30 pacientes, se dividió en dos grupos de 15 personas cada uno. Esta división se realizó de forma en que las primeras 15 personas que asistie- ron al pabellón para dicha intervención, se les administró Dexametasona $4 \mathrm{mg}$ endovenosa (EV); y las últimas 15 personas se les administro Ketoprofeno 200 mg EV previo a la operación. Cabe mencionar, que a cada grupo experimental se le aplicó, junto con los fármacos a comparar, un esquema medicamentoso quedando cada grupo distribuido de la siguiente forma:

- Grupo experimental 1: Se administró Dexametasona $4 \mathrm{mg}$, junto con Cefazolina $1 \mathrm{~g}$ y Ketorolaco $30 \mathrm{mg}$. Toda vía endovenosa 30 minutos antes de la cirugía.

- Grupo experimental 2: Se administró Ketoprofeno 200 mg, junto con Cefazolina $1 \mathrm{~g}$, ambos vía endovenosa 30 minutos antes de la cirugía.

La administración EV fue efectuada por la enfermera de turno en el pabellón y la exodoncia quirúrgica y su control postquirúrgico a los 2 y 7 días, fue realizado por los alumnos de postgrado de cirugía maxilofacial.

Medición de variables de estudio. Cada paciente del grupo 1 y 2, se controló en 3 ocasiones: Antes de la cirugía, al segundo y séptimo día postoperatorio. Para esto, se realizó la medición clínica de la inflamación utilizando el método de Neupert (Laureano Filho et al.). El cual incluye en primera instancia la palpación digital y luego el análisis visual.

Se determinaron seis puntos anatómicos en cada paciente, en ambos lados de la cara: ángulo mandibular (AM), parte más coronal del Tragus (Tr), canto externo del bulbo ocular (Cant), pliegue externo del ala de la nariz (ala). Comisura labial (Comi). Pogonion blando (Pog). Posteriormente con un hilo de sutura 3-0, sujetado con los dedos pulgar y medio, se midió la distancia entre los puntos AM - Tr (L1), AM - Cant (L2), AM - Ala (L3), AM - Comi (L4) y AM - Pog (L5), con el objetivo de trasladar dicha longitud a una regla metálica milimetrada (Fig. 1).

Se registró las medidas preoperatorias de las referencias faciales y se consignó el porcentaje de edema facial de cada medida a los 2 y 7 días posterior a la exodoncia de los puntos L1, L2, L3, L4 y L5 del lado derecho e izquierdo, restando la medida preoperatoria con la medida postquirúrgica, dividiéndola por la medida preoperatoria de cada punto respectivamente y multiplicándolo por 100 (Fig. 2), los resultados mayores a "cero" se interpretó como presencia de edema. 

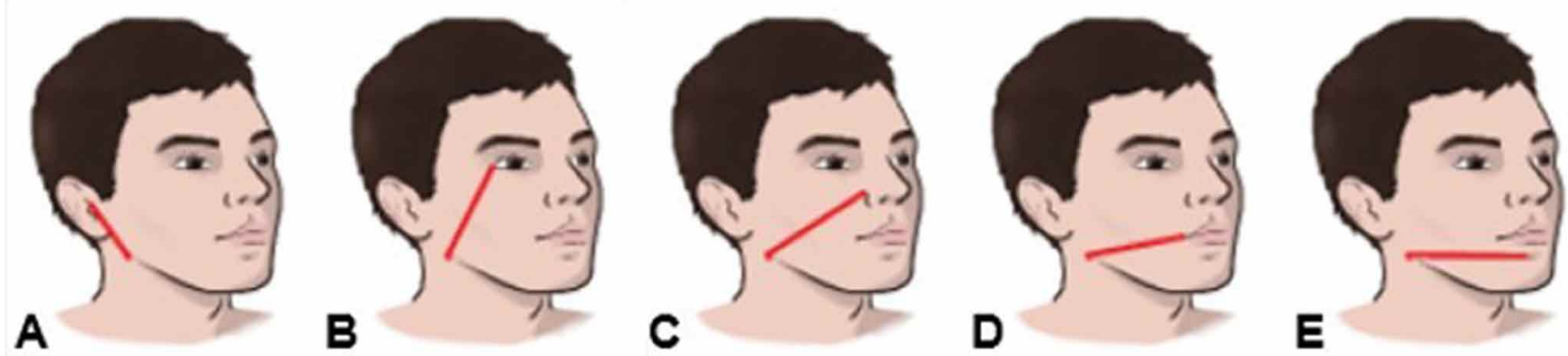

Fig. 1. Medidas del contorno facial consignadas A. Distancia ángulo mandibular a parte mas coronal del tragus (L1), B. Distancia ángulo mandibular a canto externo del ojo (L2), C. Distancia ángulo mandibular a Pliegue externo del ala de la nariz. D. Distancia ángulo mandibular a Comisura labial (L4), E. Distancia ángulo mandibular a Pogonion blando (L5)

$$
X \%=\frac{\text { (Medida preoperatoria }- \text { Medida instante de tiempo) }}{\text { Medida Preoperatoria }} \times 100
$$

Fig. 2. Fórmula para obtener porcentaje de edema facial por cada medida de longitud (L1, L2, L3, L4, L5) a los 2 y 7 días postquirúrgicos. X\%: Porcentaje de edema facial por cada medida de longitud. Medida preoperatoria: Medida Obtenida previo al procedimiento quirúrgico de cada medida de longitud. Medida instante de tiempo: Medida obtenida inmediato, 2 y 7 días posterior a la exodoncia de cada medida de longitud.

El trismus, se evaluó mediante la medición interincisal, la cual va del margen incisal del incisivo central inferior derecho al margen incisal del incisivo central superior derecho, en apertura máxima por parte del paciente. Este se obtuvo con una regla milimetrada, cuya medida se clasificó como trismus al ser igual o menor a 35 mm (Nelson et al., 1992).

El dolor, se evaluó mediante la Escala Visual Análoga (EVA), donde 0: Ausencia de dolor, 1: Dolor muy leve, 2-4: Dolor leve, 5-6: Dolor moderado, 7-8: Dolor fuerte, 9: Dolor muy fuerte y 10: Dolor insoportable.

El análisis y comparaciones de datos obtenidos se obtuvieron con el programa STATA 14.2, utilizando test Shapiro Wilk para ver la distribución de los datos, y según esta distribución se ocupó la prueba t Student, el test de Mann-Whitney y el análisis de recesión línea.

\section{Protocolo Quirúrgico Perioperatorio}

Preoperatorio. Los pacientes fueron citados $30 \mathrm{mi}-$ nutos antes de la cirugía, antes de ingresar a pabellón se les explicó el estudio y se entregó el consentimiento informado para consignar si poseen los requisitos de inclusión para ser parte del estudio.
Se completó una ficha clínica de cada sujeto, en donde fueron registrados: antecedentes personales, mediciones de las 5 líneas faciales iniciales, además de la apertura bucal inicial y se consultará si es que presenta dolor, utilizando la escala EVA.

Una vez ingresado a pabellón la enfermera, administró los fármacos según sea el caso. Los pacientes del grupo 1, se administró Dexametasona $4 \mathrm{mg}$, junto con Cefazolina $1 \mathrm{~g}$ y Ketorolaco $30 \mathrm{mg}$, todos vía endovenosa 30 minutos antes de la cirugía. Al grupo 2 se administró Ketoprofeno 200 mg, junto con Cefazolina $1 \mathrm{~g}$, ambos vía endovenosa $30 \mathrm{~min}$ antes de la cirugía.

Intraoperatorio. El procedimiento quirúrgico consistió en la exodoncia de los terceros molares inferiores incluidos que requerían osteotomía y/o odontosección.

La preparación del paciente y campo quirúrgico se realizó respetando las normas universales de control de infecciones, previa a la intervención, se realizó antisepsia oral con gluconato de clorhexidina $0,12 \%$ durante 60 segundos.

La intervención fue realizada por alumnos del postgrado de Cirugía y Traumatología Bucomaxilofacial.

Se realizó el procedimiento bajo anestesia local, Mepivacaína $2 \%$ con epinefrina $(1: 100.000)$ técnica Spix indirecta, 2 tubos $(1.8 \mathrm{ml} \mathrm{c} / \mathrm{u})$ por lado, colgajo Semi Newman con descarga distal con bisturí frío. La osteotomía se realizó con motor y fresa de carburo de tungsteno redonda $\mathrm{N}^{\circ} 12$ y constante irrigación. En caso de requerir odontosección, esta se realizó con fresa cilíndrica de carburo de tungsteno $\mathrm{N}^{\circ} 12$ y constante irrigación. La luxoavulsión dentaria se realizó con elevador mediano. 
COLLAZOS, P. C.; FONSECA. E. D.; PARADA, F. F. \& MONTERO, R. S. Estudio comparativo de la efectividad antiinflamatoria postoperatoria al usar Dexametasona endovenoso versus Ketoprofeno endovenoso previo a la exodoncia de terceros molares mandibulares incluidos. Int. J. Odontostomat., 15(4):1060-1070, 2021.

Se irrigó el lecho quirúrgico con suero fisiológico, curetaje mecánico y control de hemostasia. La reposición del colgajo se realizó con sutura seda 3-0 punto simple.

Post operatorio. A todos los pacientes se les entregó indicaciones orales y escritas acerca de los cuidados post operatorios, de no utilizar otro medicamento que no sea el prescrito y el uso apropiado de compresas de frío local las primeras 24 horas. Se recetó un esquema medicamentoso post quirúrgico, vía oral, para el control del dolor, el cual constaba de un comprimido de Ketorolaco $10 \mathrm{mg}$ cada 8 horas por 3 días.

Se citó a controles a los 2 días y 7 días postoperatorios, donde se consignó el porcentaje de edema facial de cada medida a los 2 y 7 días posterior a la exodoncia de los puntos L1, L2, L3, L4 y L5 del lado derecho e izquierdo. También se consignó la intensidad del dolor postquirúrgico con la escala de EVA y la presencia o ausencia de trismus, a los 2 y 7 días post operatorio.

\section{RESULTADOS}

La distribución de la población participante según sexo fue de 12 hombres (40\%) y 18 mujeres (60 $\%$ ), el promedio de la edad de los sujetos del estudio fue 23,3 \pm 3,334 años, con un valor mínimo de 18 años y un valor máximo de 29 años. Se presentó una diferencia estadísticamente significativa solo en 2 mediadas ( $p=0,046$ y $p=0,049)$, de las 5 evaluadas, en el porcentaje de edema a las 7 días postoperatorias en- tre ambos grupos experimentales, siendo mayor el edema en el grupo Dexametasona. A los 2 días no hubo diferencia estadística entre los grupos experimentales, debido a que ninguna " $P$ " fue menor que 0,05 , en todas las medidas analizadas (Tabla I).

En relación al dolor a todos los sujetos del estudio se les realizó una encuesta correspondiente a la intensidad del dolor medido en EVA a los 0,5, 1, 2 y 7 días después de la cirugía, en donde se comparó la intensidad del dolor en ambos grupos experimentales y en los días postoperatorios evaluados. Se determinó que no existía una diferencia estadísticamente significativa, en la intensidad del dolor a los 0,5, 1 y 2 días después según el medicamento utilizado (Fig. 3), debido a "p" no fue menor a 0,05.

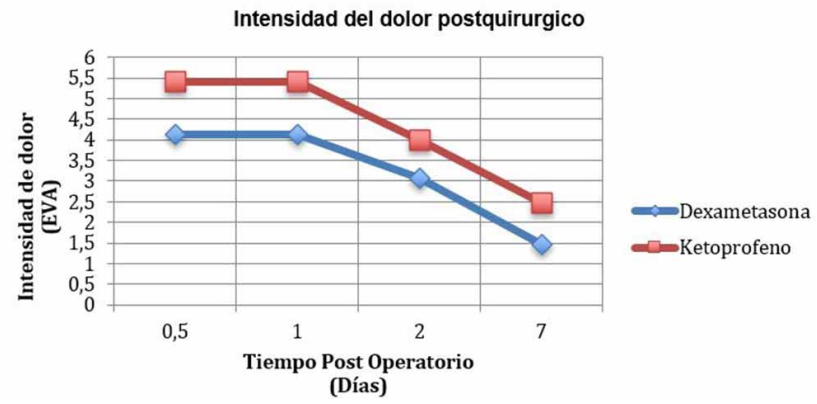

Fig. 3. Intensidad de dolor en los 0,5,1,2 y 7 días según grupos experimentales.

Sin embargo, a los 7 días se observó una diferencia estadísticamente significativa $(p=0,029)$ (Fig. 4) dando un mayor dolor a los sujetos medicados con Ketoprofeno $(2,47 \mathrm{~cm} \pm 1,55)$. En cuanto al trismus, se obtuvo que el promedio de la apertura bucal al se-

Tabla I. Promedio de porcentaje de edema facial, de cada medida,comparando ambos medicamentos para determinar su eficacia.

\begin{tabular}{clccccc}
\hline DIAS & MEDIDAS & Media & $\begin{array}{c}\text { Dexametasona 4 mg (15 sujetos) } \\
\text { Desviación } \\
\text { Estándar }\end{array}$ & Media & $\begin{array}{c}\text { Ketoprofeno } 200 \text { mg (15 sujetos) } \\
\text { Desviación } \\
\text { Estándar }\end{array}$ & $\begin{array}{c}\text { Test de Mann- } \\
\text { Whitney valor de }\end{array}$ \\
\hline Am - Tr & 2,92 & 3,57 & 3,62 & 6,40 & 0,8780 \\
2 & Am - Cant & 3,02 & 2,85 & 2,08 & 2,71 & 0.2539 \\
& Am - Ala & 7,91 & 7,60 & 8,04 & 2,33 & 0,1250 \\
& Am - Comi & 9,29 & 7,60 & 9,63 & 5,95 & 0,6940 \\
& Am - Pog & 9,01 & 4,48 & 8,90 & 7,01 & 0.7244 \\
& Am - T & 0,96 & 1,49 & 2,45 & 4,25 & 0.2239 \\
Am - Cant & 1,65 & 2,26 & 0,40 & 0,81 & 0.0461 \\
Am - Ala & 3,85 & 5,59 & 1,28 & 1,74 & 0.0490 \\
& Am - Comi & 3,80 & 4,15 & 1,85 & 1,57 & 0.1815 \\
& Am - Pog & 2,70 & 2,38 & 2,29 & 3,42 & 0.2669 \\
\hline
\end{tabular}


COLLAZOS, P. C.; FONSECA. E. D.; PARADA, F. F. \& MONTERO, R. S. Estudio comparativo de la efectividad antiinflamatoria postoperatoria al usar Dexametasona endovenoso versus Ketoprofeno endovenoso previo a la exodoncia de terceros molares mandibulares incluidos. Int. J. Odontostomat., 15(4):1060-1070, 2021.

Dolor al séptimo dia postoperatorio: Dexametasona v/s Ketoprofeno

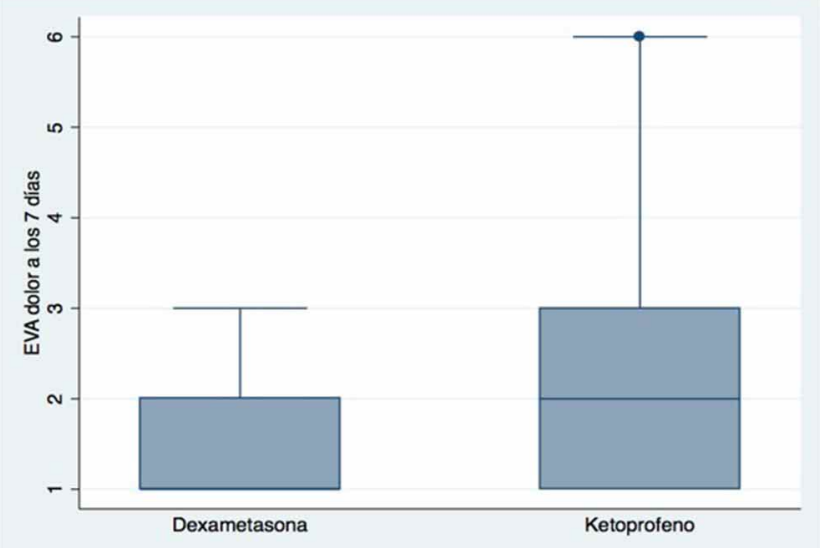

Fig. 4. Comparación de la intensidad del dolor al séptimo día entre ambos fármacos.

gundo día postoperatorio fue mayor en el grupo que utilizó Dexametasona $(27,73 \mathrm{~mm} \pm 8,60)$, en comparación al grupo que usó Ketoprofeno $(26,53 \mathrm{~mm} \pm$ $6,81)$, sin embargo, no se generó una diferencia estadísticamente significativa $(p=0,675)$.

Por otra parte, el promedio de apertura bucal evaluado a los 7 días fue mayor en el grupo Ketoprofeno $(37,2 \mathrm{~mm} \pm 6,19)$ comparado con el grupo Dexametasona $(35,8 \mathrm{~mm} \pm 7,93)$, sin ser también una diferencia estadísticamente significativa $(p=0,594)$.

En las otras variables medidas, tenemos la presencia de odontosección en la cirugía, esta generó una diferencia estadísticamente significativa, entre los sujetos que no se les realizo dicha acción versus los que si se les realizo, siendo mayor el edema en los que si se les realizo odontosección. Esta diferencia se vio principalmente en el grupo Ketoprofeno evaluado a los 2 días postoperatorios $(p=0,0248)$ (Tabla II).
Finalmente se evaluó también el uso de compresas y la duración de las cirugías con respecto a si generaban mayor porcentaje de edema inflamatorio o no, dando resultados sin diferencia estadísticamente significativa en ambos. Al realizar el análisis de regresión lineal para comparar la duración de la cirugía con el porcentaje de edema facial, los resultados a los 2 días postoperatorios indicaron que no existía una diferencia estadísticamente significativa en cuanto a la duración de la cirugía y el aumento del edema facial $(p=0,278)$, esto quiere decir que a pesar de que hubo un aumento en el edema facial a mayor duración de la cirugía, este no fue significativo (Fig. 5).

Al comparar ambas pendientes de duración y edema entre ambos grupos experimentales, tampoco se observó una diferencia estadística $(p=0,937)$ a pesar de que el edema fue aumentado más en el grupo que utilizó Dexametasona a medida que aumentaba la duración de la cirugía (Fig. 5).

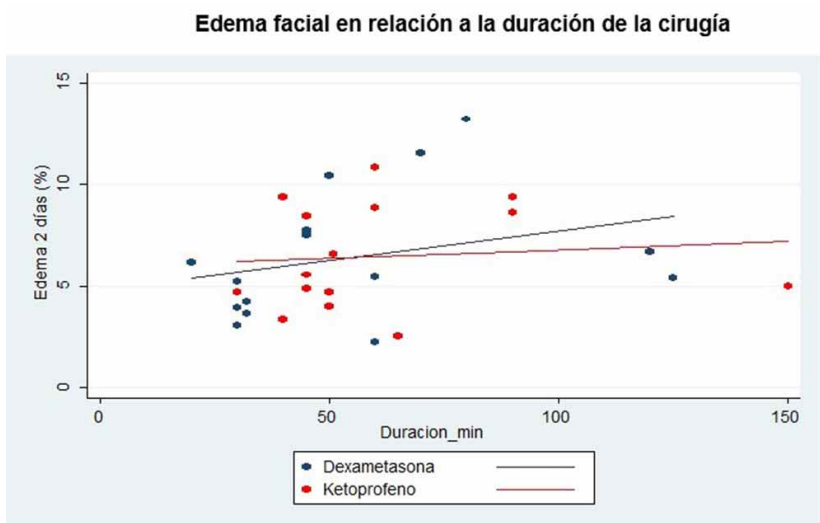

Fig. 5 Porcentaje global de edema facial a los días postoperatorios versus la duración de la cirugía, comparando ambos grupos experimentales.

Tabla II. Resumen de los promedios de porcentaje global del edema facial, alos 2 y 7 días postoperatorios en las cirugías con y sin odontosección.

\begin{tabular}{|c|c|c|c|c|c|c|c|c|}
\hline DÍAS & FARMACO UTILIZADO & GRUPO & $\begin{array}{l}\text { NÚMERO } \\
\text { SUJETOS }\end{array}$ & DE & MEDIA & $\begin{array}{l}\text { STD. } \\
\text { DESV. }\end{array}$ & $\mathrm{T}$ & $\mathrm{P}$ \\
\hline \multirow{4}{*}{2} & DEXAMETASONA & NO & 7 & & 4,86 & 1,58 & \multirow[t]{2}{*}{$-1,9588$} & \multirow[t]{2}{*}{0,0719} \\
\hline & & SI & 8 & & 7,81 & 3,69 & & \\
\hline & \multirow[t]{2}{*}{ KETOPROFENO } & NO & 6 & & 4,69 & 0,72 & \multirow[t]{2}{*}{$-2,5368$} & \multirow[t]{2}{*}{0,0248} \\
\hline & & SI & 9 & & 7,63 & 2,74 & & \\
\hline \multirow{4}{*}{7} & DEXAMETASONA & NO & 7 & & 2,01 & 1,53 & \multirow[t]{2}{*}{$-1,2192$} & \multirow[t]{2}{*}{0,2444} \\
\hline & & SI & 8 & & 3,10 & 1,89 & & \\
\hline & \multirow[t]{2}{*}{ KETOPROFENO } & NO & 6 & & 0,91 & 0,67 & \multirow[t]{2}{*}{$-2,0470$} & \multirow[t]{2}{*}{0,0614} \\
\hline & & SI & 9 & & 2,15 & 1,37 & & \\
\hline
\end{tabular}


COLLAZOS, P. C.; FONSECA. E. D.; PARADA, F. F. \& MONTERO, R. S. Estudio comparativo de la efectividad antiinflamatoria postoperatoria al usar Dexametasona endovenoso versus Ketoprofeno endovenoso previo a la exodoncia de terceros molares mandibulares incluidos. Int. J. Odontostomat., 15(4):1060-1070, 2021.

\section{DISCUSIÓN}

Existe numerosa bibliografía acerca de los fármacos que se utilizan para mejorar el postoperatorio de la cirugía de los terceros molares, sin embargo, la evidencia demuestra que el uso de corticoides, especialmente la Dexametasona, constituye la primera elección de varios autores, debido a su acción netamente glucocorticoide y nula acción mineralcorticoide (Alexander \& Throndson, 2000; Kim et al., 2009). Por otro lado, la premedicación con AINES para el manejo del dolor y edema facial postquirúrgico, a sido ampliamente estudiado y en muchos casos aceptada como protocolos prequirúrgicos en cirugía oral. En relación con las características personales de cada paciente, se encuentra el género y edad, en donde la mayor parte de los estudios reportan una distribución proporcional entre hombres y mujeres. Tal es el caso de los estudios de Majid \& Mahmood, en el cual hubo 16 hombres y 14 mujeres; Grossi et al. también presentaron una distribución de género proporcional a lo antes descrito, ya que se presentaron 6 hombres y 9 mujeres, para cada grupo experimental, dando un total de 12 hombres y 18 mujeres.

El promedio de la edad presentado en este estudio fue de 22,87 años $\pm 3,270$ y 23,73 años \pm 3,453 para el grupo Dexametasona y Ketoprofeno respectivamente. Esto coincide con los estudios de Kumar et al., Laureano Filho et al., y Ustün et al. (2003) los cuales exponen promedios de edad entre los $20-25$ años, similar a lo sucedido en este estudio.

Al comparar el promedio del porcentaje de edema en cada una de las medidas faciales evaluadas, tanto a los 2 y 7 días postoperatorias entre los sujetos que utilizaron Dexametasona y que usaron Ketoprofeno, es importante mencionar que no existen estudios que analicen estas situaciones, pues son múltiples las variables que inciden en la mayor o menor producción de edema.

En el caso de los que usaron Ketoprofeno, se presume que este grupo experimental debía presentar más edema facial en todas las medidas, puesto que los AINES tienen su efecto primario al inhibir la acción de la enzima ciclooxigenasa (tanto la COX-1 como la COX-2), la cual es la responsable de convertir el ácido araquidónico en variados productos pertenecientes al grupo llamado prostanoide, como las: prostaglandinas, tromboxanos y prostaciclinas, las cuales juegan un rol fundamental en la homeostasis celular como también en la mediación de la respuesta inflamatoria (Furse et al., 2006; Yao et al., 2007). Los corticoides actúan a nivel de la enzima fosfolipasa A, la cual es la encargada de convertir los fosfolípidos de la membrana celular en acido araquidónico, por lo que al inhibir esta enzima, no se podrá generar este acido, y tanto la ciclooxigenasas como las lipooxigenasas no podrán convertir este acido en prostaglandinas $y$ leucotrienos correspondientemente. Estos mediadores químicos últimos son sustancias hipersensibilizantes y vasoconstrictoras, que junto a las prostaglandinas son importantes mediadores del proceso inflamatorio y serán responsables de las manifestaciones clínicas de la inflamación (Majid \& Mahmood; Yao et al.). Mientras más arriba la cascada de la inflación esté inhibida, menor serán los mediadores químicos inflamatorios que se producen, por lo que se generara menos dolor y edema. Contrariamente a lo anterior, los resultados de este estudio arrojaron un mayor porcentaje de edema en dos medidas faciales controladas a los 2 días postquirúrgicos, presentes en Am-Cant y Am-Pog, donde la mayoría de las medidas faciales registradas a los 7 días fueron mayores en los sujetos que se les administro Dexametasona, salvo en la medida Am-Tr, aún así, esta diferencia de edema presentado en ambos grupos experimentales y registrado en 5 medida, no resultó ser estadísticamente significativa $(p<0,05)$, salvo en 2 medida evaluadas a los 7 días.

Otro de los análisis fueron los promedios del porcentaje global de edema facial, a los 2 y 7 días postoperatorios en las cirugías con o sin odontosección, con ambos medicamentos. Se destaca una diferencia estadísticamente significativa ( $p$ de 0,0248 ) en el promedio del porcentaje de edema facial a los 2 días postoperatorios entre el grupo que si se le realizo odontosección y al que no se le realizo esta intervención, siendo mayor el edema facial en los sujetos que utilizaron Ketoprofeno. Esto quiere decir, que el edema fue significativamente mayor en los pacientes que se les realizo la odontosección versus los que no se les realizo esta acción. Por otra parte, se observó una tendencia a la diferencia estadísticamente significativa ( $p$ de 0,0719 y $p$ de $0,0614)$ en las demás variables evaluadas, esto se condice con lo relatado por diferentes autores. Según Lombardía et al. (1989) el cual menciona que la odontosección y la osteotomía aumentan el dolor y 
COLLAZOS, P. C.; FONSECA. E. D.; PARADA, F. F. \& MONTERO, R. S. Estudio comparativo de la efectividad antiinflamatoria postoperatoria al usar Dexametasona endovenoso versus Ketoprofeno endovenoso previo a la exodoncia de terceros molares mandibulares incluidos. Int. J. Odontostomat., 15(4):1060-1070, 2021.

edema tras la intervención quirúrgica. También, Estarelles et al. (2000) menciona que el dolor y edema postquirúrgico en su estudio, aumentó significativamente con la odontosección, el tiempo de osteotomía y el tiempo total de la intervención, además menciona que el edema tras la extracción del tercer molar es una reacción fisiológica inevitable y está relacionada directamente con el traumatismo quirúrgico, a pesar de ser un efecto secundario observable (Sánchez et al., 1992). En relación al promedio de porcentaje global de edema facial a los 2 días y a los 7 días después de la cirugía.

Respecto al uso o no de compresas de frío las primeras 24 horas, autores como Pérez et al. (2016) mencionan que el uso de crioterapia tiene un efecto comprobado en reducción de edema y dolor postoperatorio en este tipo de cirugías, sin embargo, su beneficio depende de la correcta adhesión y compromiso del paciente a la terapia, por lo que se considera una terapia complementaria. En nuestro caso, el análisis determino que no existía una diferencia estadísticamente significativa en que el uso de compresas las primeras 24 horas sería un factor que disminuye el porcentaje de edema facial, en ambos grupos experimentales y días evaluados, debido a que ningún "p" fue menor a 0,05. Esto lo atribuimos a lo mencionado por van der Westhuijzen et al. (2005) que relata una falta de compromiso por el paciente de seguir la instrucción de cómo realizar el uso de compresas de frío local.

En este estudio, aparte de considerar el edema, también se analizaron las variables dolor y trismus, donde la comparación de la intensidad del dolor entre los grupos experimentales, se demostró que no existía una diferencia estadísticamente significativa en la mayoría de los días evaluados, donde hay una diferencia de a lo menos $1 \mathrm{~cm}$ entre el grupo Dexametasona y Ketoprofeno, siendo mayor la intensidad de dolor en los pacientes que se les administro el Ketoprofeno, aún así, esto no fue suficiente para lograr ser una diferencia estadística, esto se debe por ejemplo, a lo reportado por Markiewicz et al. (2008) en donde confirmó que los corticoides no disminuyeron el dolor con diferencias estadísticamente significativas con respecto al grupo control y destacó que el dolor, al ser subjetivo, se ve influenciado por múltiples factores (edad, sexo, ansiedad y la dificultad quirúrgica).

Se presentó una diferencia estadísticamente significativa $(p=0,028)$, entre los grupos experimen- tales, que se evaluaron a los 7 días, dando una mayor intensidad de dolor en los pacientes que se utilizó Ketoprofeno $(2,47 \mathrm{~cm} \pm 1,55)$. En el mismo sentido que el presente estudio, Orellana \& Salazar (2007), en su investigación para evaluar y comparar la Dexametasona con Ibuprofeno y un placebo, en el control de la inflamación y dolor debido a la cirugía de los terceros molares, reportaron disminución significativa del dolor e inflamación al administrar Dexametasona, en comparación al Ibuprofeno.

El trismus, que por lo general no se ve influenciado por los Corticoides ni AINES, los estudios de Grossi et al. y Ustün et al. no encontraron diferencias significativas en relación al trismus generado en ninguno de los días postoperatorios que cada autor evaluó, versus los medicamentos que utilizaron, sin embargo, Moore et al. (2005) reportaron una mayor efectividad de la Dexametasona como estrategia terapéutica para limitar el trismus, al compararla con una dosis preoperatoria de un inhibidor selectivo de la COX-2 como el Rofecoxib. En particular, en este estudio no se observó una diferencia estadísticamente significativa en la generación trismus, siendo que al segundo día los pacientes que se administró Dexametasona presentaron menor trismus que los que se les administro Ketoprofeno, aunque este resultado fue al revés, al evaluarlo al séptimo día, aún así, no se logró generar una diferencia estadística, debido a que los 2 medicamentos ayudaron en el control de este proceso. Cabe señalar que no es extraño que la Dexame-tasona no influya en el trismus de forma significativa, ya que se presume que la relación entre ellos recae en un supuesto efecto secundario de la acción antiinflamatoria en la apertura bucal, pero no está del todo comprobado (Orellana \& Salazar).

Para finalizar, se realizó un análisis para ver si la duración de la intervención quirúrgica producía un efecto en la generación de un mayor o menor edema facial, y además se comparó esto entre los 2 grupos experimentales. Muchos investigadores refieren un mayor dolor y tumefacción a mayor duración de la intervención, puesto que la reacción fisiológica del cuerpo será más exagerada al perdurar el trauma quirúrgico por mayor tiempo, es por esto que en éste tipo de cirugía comúnmente se espera que el sistema inmune del cuerpo humano reconozca esta acción como un ataque, por lo tanto active las diferentes cascadas de la inflamación para controlar el daño y se produzca un edema desproporcional (Grossi et al.; Estarelles et al.; Pérez et al.). 
Según los resultados que se obtuvieron en esta investigación, se concluye que si bien, hubo un aumento en el promedio del porcentaje global de edema facial a mayor tiempo operatorio, éste no fue estadísticamente significativo, evaluado tanto a los 2 días como a los 7 días. También, al comparar los grupos experimentales, respecto a cuál de los dos generó mayor edema facial que el otro, a favor del tiempo operatorio, se observa una diferencia a los 2 días postoperatorios que no es estadísticamente significativa $(p=0,937)$. Este resultado puede deberse a otros factores implícitos en la cirugía, ya sea como el uso o no de odontosección, la habilidad del operador y otras variables que pueden alargar o acortar el acto quirúrgico, haciendo que se genere más o menos edema inflamatorio.

\section{CONCLUSIÓN}

El uso de Dexametasona $4 \mathrm{mg}$, administrado vía endovenosa, previo a la cirugía de terceros molares incluidos genera menos porcentaje de inflamación, medido a los 2 días post operatorios, en la mayoría de las cinco medidas analizadas, en comparación a los sujetos que se les administro Ketoprofeno $200 \mathrm{mg}$. Sin embargo, no presentan una diferencia estadísticamente significativa, por lo que no se justifica el uso especifico de Dexametasona 4 $\mathrm{mg}$ para el control del edema, debido a que ejercen el mismo efecto antiinflamatorio ambos fármacos.

En cuanto al uso de Ketoprofeno, administrado vía endovenosa, genera menos porcentaje de edema facial, en la mayoría de las medidas analizadas a los 7 días postoperatorios, en comparación con Dexametasona. En este caso dos de las cinco medidas, presentaron una diferencia estadísticamente significativa, pero aún así no genera una diferencia global entre el grupo que se administró Dexametasona, por lo que su uso no mejorara más la hinchazón facial en comparación a Ketoprofeno.

El trismus y el uso de compresas las 24 horas postcirugía no se ven afectados por el uso de Dexametasona o Ketoprofeno, aunque se genere un menor trismus a los 2 días postoperatorios en los pacientes que se utilizó Dexametasona $4 \mathrm{mg}$, la muestra no fue la suficiente para determinar una diferencia significativa.

Los pacientes que se les administro
Dexametasona $4 \mathrm{mg}$. Dieron una tendencia a genera una diferencia significativa en la formación de edema con respecto al grupo que se le realizo odontosección versus el que no. Esto nos hace concluir que, la Dexametasona $4 \mathrm{mg}$. puede influenciar de manera negativa en la formación del edema en cirugías con odontosección, por lo que generó menos diferencia de hinchazón comparado con los sujetos que se utilizó Ketoprofeno 200 mg, por lo que se recomienda el uso de Dexametasona $4 \mathrm{mg}$, vía endovenosa en cirugías que se deba realizar odontosección.

El uso de Dexametasona $4 \mathrm{mg}$, va a influenciar tanto en el dolor postoperatorio, como en la formación de edema facial en cirugías de mayor duración, en comparación al Ketoprofeno 200 mg, aunque no de forma significativa, aún así, se recomienda su uso por sobre el otro fármaco, esto es debido, a que la mayor intensidad de dolor postoperatorio se presentó en los sujetos que se les administro Ketoprofeno $200 \mathrm{mg}$ endovenoso, teniendo una diferencia significativa en la intensidad, entre los que utilizaron Dexametasona al medirlo a los 7 días postoperatorios. Cabe destacar que a ambos grupos experimentales se les dio a seguir el mismo esquema medicamentoso postoperatorio, vía oral, por lo que, este factor no influyo al momento de medir la intensidad del dolor.

Se observó un aumento del edema facial a medida que duraban más las intervenciones quirúrgicas, acentuándose este edema en los pacientes que utilizaron Dexametasona $4 \mathrm{mg}$, la diferencia con los pacientes que utilizaron Ketoprofeno 200mg es muy leve y no llega a ser significativa, por lo que el uso de cualquiera de estos dos antiinflamatorios está bien indicado.

Dentro de las limitaciones del estudio se encuentran la ausencia del ciego de los pacientes, pues éstos conocían su premedicación, lo cual puede falsear la perspectiva de éstos en cuanto al dolor, también está la muestra, la cual fue calculada de forma no probabilística al azar, pero con datos reales obtenidos de los estudios previos, pero estimando sólo variables que incluían edema.

Para las próximas investigaciones se sugiere considerar la necesidad de aumentar el tamaño muestral, para así lograr inferir estadísticamente en las variables que también forman parte de la respuesta postoperatoria en estas cirugías. 
COLLAZOS, P. C.; FONSECA. E. D.; PARADA, F. F. \& MONTERO, R. S. Comparative study of postoperative antiinflammatory effectiveness when using intravenous Dexamethasone versus intravenous Ketoprofen prior to extraction of mandibular third molars included. Int. J. Odontostomat., 15(4):1060-1070, 2021.

ABSTRACT: The extraction of included third molars most of the time involves an acute inflammatory picture, postoperative pain and trismus. At present, various pharmacological protocols have been proposed in order to prevent these complications, where the most widely used are analgesics and corticosteroids. Compare Dexamethasone and Ketoprofen used intravenously prior to surgery of mandibular third molars included, in the control of edema, pain and trismus. An experimental, analytical, crosssectional study was carried out, authorized by the ethics committee of the Andrés Bello University. Thirty subjects who required extraction of included mandibular third molars were chosen, 15randomly received intravenous Dexamethasone $4 \mathrm{mg}$ and 15 intravenous Ketoprofen $200 \mathrm{mg} 30$ minutes before the intervention. The surgical procedure was standardized, facial edema, trismus and postoperative pain were evaluated at 2 and 7 days after the surgery. There was a statistically significant difference only in 2 facial measures, of the 5 taken, when comparing the percentage of edema at 7 postoperative days between both experimental groups. Most of the Dexamethasone group had a larger volume edema compared to the Ketoprofen group. In relation to pain and trismus, significant differences were only observed in the intensity of pain evaluated at 7 days, being greater in the Ketoprofen group. In the other variables measured, the presence of a dental section in the surgery generated a statistically significant difference, with the edema being greater in those who underwent a dental section. This difference was mainly seen in the Ketoprofen group evaluated at 2 postoperative days. The use of any of the 2 drugs is well indicated to alleviate the pain and inflammation of the patient generated by the trauma caused by this type of surgery. Although the group treated with Dexamethasone presented less pain, it was not a significant difference compared to the group with Ketoprofen.

KEY WORDS: dexamethasone, ketoprofen, edema, third Molar, trismus

\section{REFERENCIAS BIBLIOGRÁFICAS}

Alexander, R. E. \& Throndson, R. R. A review of perioperative corticosteroid use in dentoalveolar surgery. Oral Surg. Oral Med. Oral Pathol. Oral Radiol. Endod., 90(4):406-15. 2000.

Buesa Martínez, O. Estudio Comparativo de Estabilidad Fisicoquímica y Disponibilidad In vitro de Ketoprofeno en Formas Tópicas Desarrolladas con Nuevos Excipientes. Tesis de Doctorado. Madrid, Universidad Complutense de Madrid, Facultad de Farmacia, Departamento de Farmacia y Tecnología Farmacéutica I, 1995. Disponible en: https://eprints.ucm.es/id/ eprint/3906/
Büyükkurt, M. C.; Tozoglu, S.; Aras, M. H. \& Yolcu, U. Ectopic eruption of a maxillary third molar tooth in the maxillary sinus: a case report. J. Contemp. Dent. Pract., 6(3):104-10, 2005.

de la Cruz Carranza, H.; Asmat, Á. S. \& Guerrero, R. Efectividad del tratamiento profiláctico con dexametasona de 8 y $4 \mathrm{mg}$ para controlar el edema poscirugía de terceros molares incluidos: ensayo clínico aleatorizado de grupos en paralelo. Rev. Esp. Cir. Oral Maxilofac., 35(4):157-61, 2013.

Estarelles, R.; Gay, C. \& Bagán, J. V. Estudio del postoperatorio tras la extracción quirúrgica de 100 terceros molares inferiores impactados en relación a la dificultad del acto. Av. Odontoestomatol., 16(6):377-82, 2000.

Esteller M. V.; Paredes G. J.; Valmaseda C. E.; Berini A. L. \& GayEscoda, C. Eficacia analgésica del diclofenaco sódico vs. ibuprofeno después de la extracción quirúrgica de un tercer molar inferior incluido. Med. Oral Patol. Oral Cir. Bucal, 9(5):444-53, 2004.

Furse, K. E.; Pratt, D. A.; Schneider, C.; Brash, A. R.; Porter, N. A. \& Lybrand, T. P. Molecular dynamics simulations of arachidonic acid-derived pentadienyl radical intermediate complexes with COX-1 and COX-2: insights into oxygenation regio- and stereoselectivity. Biochemistry, 45(10):3206-18, 2006.

García, C.; Collado, J.; Monje, F., Serrano, H.; Morillo, A. J.; Mateo, J. \& Quirós, P. Tercer molar ectópico a nivel de región infraorbitaria-seno maxilar. Rev. Esp. Cir. Oral Maxilofac., 29:3, 2007.

Gay-Escoda, C. \& Berini, A. L. Tratado de Cirugía Bucal. Tomo I. Madrid, Ergón, 2004.

Grossi, G. B.; Maiorana, C.; Garramone, R. A.; Borgonovo, A.; Beretta, M.; Farronato, D. \& Santoro, F. Effect of submucosal injection of dexamethasone on postoperative discomfort after third molar surgery: a prospective study. J. Oral Maxillofac. Surg., 65(11):2218-26, 2007.

Herrera-Briones, F. J.; Prados S. E.; Reyes B. C. \& Vallecillo C. M. Update on the use of corticosteroids in third molar surgery: systematic review of the literature. Oral Surg. Oral Med. Oral Pathol. Oral Radiol., 116(5):e342-51, 2013.

Kim, K.; Brar, P.; Jakubowski, J.; Kaltman, S. \& Lopez, E. The use of corticosteroids and nonsteroidal antiinflammatory medication for the management of pain and inflammation after third molar surgery: a review of the literature. Oral Surg. Oral Med. Oral Pathol. Oral Radiol., 107(5):630-40, 2009.

Kumar, M.; Singla, R.; Gill, G. S.; Kalra, T. \& Jain, N. Evaluating combined effect of oral premedication with ibuprofen and dexamethasone on success of inferior alveolar nerve block in mandibular molars with symptomatic irreversible pulpitis: a prospective, double-blind, randomized clinical trial. J. Endod., 47(5):705-10, 2021.

Laureano Filho, J. R.; Maurette, P. E.; Allais, M.; Cotinho, M. \& Fernandes, C. Clinical comparative study of the effectiveness of two dosages of Dexamethasone to control postoperative swelling, trismus and pain after the surgical extraction of mandibular impactedthird molars. Med. Oral Patol. Oral Cir. Bucal, 13(2):E129-32, 2008.

Lombardía, E; González, M; Junquera, L. M. \& Gener, M. Estudio del dolor postoperatorio habido tras la extracción de terceros molares inferiores. Rev. Esp. Cir. Oral Maxilofac., 11:98-104, 1989.

Majid, O. W. \& Mahmood, W. K. Effect of submucosal and intramuscular dexamethasone on postoperative sequelae after third molar surgery: comparative study. Br. J. Oral Maxillofac. Surg., 49(8):647-52, 2011.

Markiewicz, M. R.; Brady, M. F.; Ding, E. L. \& Dodson, T. B. Corticosteroids reduce postoperative morbidity after third molar surgery: a systematic review and meta-analysis. J. Oral Maxillofac. Surg., 66(9):1881-94, 2008. 
COLLAZOS, P. C.; FONSECA. E. D.; PARADA, F. F. \& MONTERO, R. S. Estudio comparativo de la efectividad antiinflamatoria postoperatoria al usar Dexametasona endovenoso versus Ketoprofeno endovenoso previo a la exodoncia de terceros molares mandibulares incluidos. Int. J. Odontostomat., 15(4):1060-1070, 2021.

Moore, P. A.; Brar, P.; Smiga, E. R. \& Costello, B. J. Preemptive rofecoxib and dexamethasone for prevention of pain and trismus following third molar surgery. Oral Surg. Oral Med. Oral Pathol. Oral Radiol. Endod., 99(2):E1-7, 2005.

Moraschini, V.; Hidalgo, R. \& Porto B. E. Effect of submucosal injection of dexamethasone after third molar surgery: a metaanalysis of randomized controlled trials. Int. J. Oral Maxillofac. Surg., 45(2):232-40, 2016.

Nelson, S. J.; Nowlin, T. P. \& Boeselt, B. Consideration of linear and angular values of maximum mandibular opening. Compendium (Newtown, Pa.), 13(5):362-6, 1992.

Orellana, A. \& Salazar, E. Evaluación clínica de la terapia con dexametasona e ibuprofeno en la cirugía de los terceros molares retenidos. Acta Odontol. Venez., 45(1):54-60, 2007.

Pérez, H.; Bravo, R.; Mardones, M. \& Argandoña, J. Estudio comparativo en la utilización de drenaje postextracción de terceros molares impactados. Rev. Esp. Cir. Oral Maxilofac., 38(2):70-5, 2016.

Prieto, I.; Prieto-Fenech, A. \& Bascones, A. Corticoesteroides y cirugía del tercer molar inferior: Revisión de la literatura. $A v$. Odontoestomatol., 21(5):251-8, 2005.

Salmerón, J. I.; del Amo, Á.; Plasencia, J.; Pujol, R. \& Vila, C. N. Ectopic third molar in condylar region. Int. J. Oral Maxillofac. Surg., 37(4):398-400, 2008.

Sánchez, M. Análisis del Dolor, Tumefacción y Trismo después de la Exodoncia del Tercer Molar Inferior Retenido, en Relación con la Edad, el Sexo de los Pacientes, y con la Dificultad de la Técnica Quirúrgica. Madrid. Tesis de Doctorado. Madrid, Universidad Complutense de Madrid, Facultad de Medicina, 1992.

Ustün, Y.; Erdogan, O.; Esen, E. \& Karsli, E. D. Comparison of the effects of 2 doses of methylprednisolone on pain, swelling, and trismus after third molar surgery. Oral Surg. Oral Med. Oral Pathol. Oral Radiol. Endod., 96(5):535-9, 2003.

van der Westhuijzen, A. J.; Becker, P. J.; Morkel, J. \& Roelse, J. A. A randomized observer blind comparison of bilateral facial ice pack therapy with no ice therapy following third molar surgery. Int. J. Oral Maxillofac. Surg., 34(3):281-6, 2005.

Weinberg, M. \& Froum, S. J. Fármacos en odontología: Guía de prescripción. Ciudad de México, El Manual Moderno, 2014.

Yao, J. C.; Duan, W. G.; Yun, Y.; Liu, D. Q.; Yan, M.; Jiang, Z. Z. \& Zhang, L. Y. Screening method for nonsteroidal antiinflammatory drugs based on the cyclooxygenase 2 pathway activated by serum-free stimulation in A549 cells. Yakugaku Zasshi, 127(3):527-32, 2007.

\author{
Dirección para correspondencia: \\ Sandra Montero Riffo \\ Echaurren \#237 \\ Santiago \\ Región Metropolitana \\ CHILE
}

E-mail: d.fonseca@uc.cl 